\title{
3D printed patient-specific bone models for anatomy education from medical imaging
}

DOI : 10.36909/jer.ICIPPSD.15521

Arivazhagan Pugalendhi*, SenthilMurugan Arumugam, Rajesh Ranganathan, Sivakumar Ganesan

Department of Mechanical Engineering, Coimbatore Institute of Technology, Coimbatore,

Tamil Nadu, India

*Email: arivazhagan.mech02@gmail.com; Corresponding Author

\begin{abstract}
Evolution of 3D printing from medical image datasets are escalating and has widespread in healthcare applications such as anatomical models, surgical guides, and customized implants. In 3D printing, solid objects are fabricated by the frequently added the thin layers of material as per the digital model. This paper demonstrates the fabrication of 3D printed patient-specific bone models of leg and ankle foot from Digital Imaging and Communications in Medicine (DICOM) files. Processing of DICOM file is prepared by D2P (DICOM to PRINT) software and physical models are produced by Stratasys uPrint 3D printer. This 3D printed anatomical model eliminates the requirement of actual human bones, significance of preservation and mistakes in assembly of bones. The results of the study not only encourage education, surgical planning and validating medical devices but stimulate exciting innovations.
\end{abstract}

Keywords: 3D printing; medical imaging; human anatomy; medical education; surgical planning.

\section{INTRODUCTION}

Understanding the anatomical structure of the human body always remains challenging. It could be compensated by using cadaver for medical education and training purposes. It is evident that use of cadavers for dissection in anatomy learning is expensive, time consuming and potentially hazardous (Lim et al., 2016). Legal restriction for attaining the cadavers for anatomy education, preservation difficulties, demand for body donation, time constrains in body cremate and financial burden of purchasing a very accurate durable models could be solved by using $3 \mathrm{D}$ printed anatomical model in its place (AbouHashem et al., 2015; Smith et al., 2018). 3D printing is a process of fabricating the physical objects through selectively deposits the material by successive layers as per the 3D CAD (Computer Aided Design) model (Vithani et al., 2019). Stereolithography Apparatus (SLA), Fused Deposition Modelling (FDM), Multi-jet Modeling (MJM) and Digital Light Processing (DLP) are 
extensively used 3D printing techniques in medical application based on their requirement (Aimar et al., 2019). Nowadays, 3D printing is widely used in customized implants, prosthesis, surgical guides, anatomical model, surgical planning, and simulation (Ratinam et al., 2019). Significant feature of implementing 3D printing in education and training is its development of patient-specific model. Development of customized 3D printed anatomical model helps to understand unique case model for better treatment process (Panesar et al., 2019). 3D printing in healthcare application helped to overcome traditional methodology of mathematical concept to understand and investigate anatomical structure for education and training (Chen et al., 2017).

An intrinsic study of neuroanatomical structure showed that, significance in teaching and training purpose using three-dimensional physical model has proved notable improvement. A neuroanatomical study also proved that, 3D physical modeling activity was proven to improve the effectiveness for teaching spatial relationships of brain anatomy and the students performed significantly better on the understanding of periventricular structures (Drapkin et al., 2015; Estevez et al., 2010). Applications of 3D printing provides a great hike in anatomy education purpose which enhances the learning activities and better understanding of the anatomical structures than textbook images and virtual models. 3D printed anatomical models are less expensive, easy to fabricate and robust structure when compared to regular bone kits (Govsa et al., 2017; Van Epps et al., 2015).

Development of 3D printed patient-specific anatomical models comprises of three stages; namely (i) image acquisition, (ii) image processing and (iii) 3D printing (Aimar et al., 2019). Initially image acquisition is considered to be a base line step for the following process; quality of image acquired during acquisition process likely influences the outcome of the printed model (Van Eijnatten et al., 2017). Most used methods to acquire digital image datasets were Magnetic resonance imaging (MRI), Computed Tomography (CT), Positron-Emission Tomography (PET) and X-rays. Based on the required ROI (Region of Interest), CT data was identified and considered to be suitable imaging technique to capture bone model and digital images were exported in the DICOM (Digital Imaging and Communication in Medicine) file format (van Eijnatten et al., 2018). DICOM is an international standard used in imaging modalities and modeling software to communicate and manage the biomedical imaging information, data (Bidgood et al., 1997). There are several number of available medical modeling software cleared FDA (Food and Drug Administration) regulations namely D2P (DICOM to PRINT), Mimics, in Print, Vitrea, OsiriX MD and 3D Slicer (Chang et al., 2019). Fundamental function of this software is to interpolate the surface between the individual slices of scan data to form a 3D model (Yoo, 2011). 
The aim of this study is to fabricate the 3D printed patient-specific bone models by utilizing the D2P software and FDM uPrint to enhancing the medical education, training and surgical planning. It is evident that some of the most significant applications of 3D printing of an anatomical model include education, training, pathology diagnostics and patient-specific pre- and post-operative assessments ( $\mathrm{Li}$ et al., 2017). Owing to the monochromatic characteristic, hard tissue and simple in image processing, framework of this study is initially started with bone models (AbouHashem et al., 2015). FDM is most cost-effective manufacturing technology among all other 3D printing techniques with mere accuracy, this makes FDM an eligible method to adopt for medical applications especially in development of pre-surgical planning and training anatomical models (Mukul \& Vinod, 2018).

Good mechanical properties, inexpensive, ability to withstand sterilization processes and better accuracy are the key benefits of the FDM. However, removal of support material and opaque models are downside of this technology (Ganguli et al., 2018). Starosolski et al., (2014) utilizes the FDM technology to exhibits the surgical planning and simulation for pediatric musculoskeletal disorders of coxa vara deformity, Perthes disease, Blount disease and fibrous subtalar coalition. They print the models using Acrylonitrile Butadiene Styrene (ABS) material, to improvise the efficiency of accessing the pre and post-operative procedures. In another study, corrective surgery for Hallux valgus deformity is successfully operated by correction of foot alignment with the aid of 3D printed personalized models which are attained from CT images. Intraoperative navigation and complex anatomical information such as measurement, angle and structure is clearly observed and offer surgical practices prior to surgery (Ozturk et al., 2020). An attempt was made through this work to create the framework for developing patient-specific bone models for education using the medical imaging and 3D printing. This will provide the guideline for medical students, teachers, doctors, and engineers who are working in this area.

\section{METHODOLOGY}

In this study, D2P software (3D Systems, Rock Hill, United States) is used to create 3D model from obtained DICOM images. For this research purpose, anonymous CT scan data of right leg (adult female) is received from the senior orthopedic surgeon with the permission of both patient and hospital dean. D2P facilitates to segment the bone leg segment from the surrounding blood vessels and soft tissues. Initially, CT scan data was obtained with $1 \mathrm{~mm}$ slice thickness, $0.5 \mathrm{~mm}$ pixel size, $512 \times 512 \mathrm{~mm}$ pixel matrix and 16 bits per pixel is imported in D2P for further image-processing operations. Figure 1 illustrates the steps involved in image processing. Where D2P, medical 
modeling platform allows following three major image-processing operations; thresholding, region growing and model meshing.

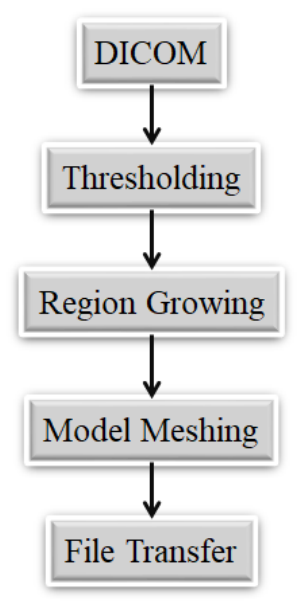

Figure 1 Steps in image processing.

Thresholding is defined as the process of selecting the anatomy from its surrounding structure based on the radio density difference among them and greyscale intensity of the scanned data (Hnatkova et al., 2014). Region growing is an intricate step in the process of segmentation that allows the user to select and delineate single anatomical structure from its surrounding (Fan et al., 2005; SoltaniNabipour et al., 2020). Although, automated algorithmic segmentation is available with the software semi-automatic segmentation approach would be best to adopt in bone segmentation technique (Wallner et al., 2018). Meshing is tessellation of triangular geometry in space domain that remain non-overlapping which also include subdivided facets on it (Byrne et al., 2016).

Data development and file format exchange are significant operations limited to the software used for medical modeling. Purpose of converting into STL (Standard Tessellation Language) file format is to divide the model into number of possible slices. Object (.obj) is a versatile file format capable of transferring information of NURBS (Non-Uniform Rational B-Splines) geometry along with object names, materials, texture and color (Iancu, 2018). STL file format possess information of surface with series of triangular facets connected without any overlap or gap between them (Zhang $\& \mathrm{Li}, 2016)$. This makes the developed model a watertight 3D model. Accuracy of the surface varies based on facet deviation, which in turn decreases accuracy with increase in facet deviation and vice versa (Bibb et al., 2015). Among the above-mentioned formats D2P software can generate standard internationally acceptable. stl and .obj file formats that are used in majority of printing technologies. In addition, D2P also allows generating individual part of a model as separate model in .stl file format which in turn supports to develop assembled parts. This helps to achieve single 
model with assembled parts especially in cases of multi-material printing. However, these exported file formats were widely used to connect 3D printer. Therefore, it helps to proceed further with $3 \mathrm{D}$ printing of the anatomical structures.

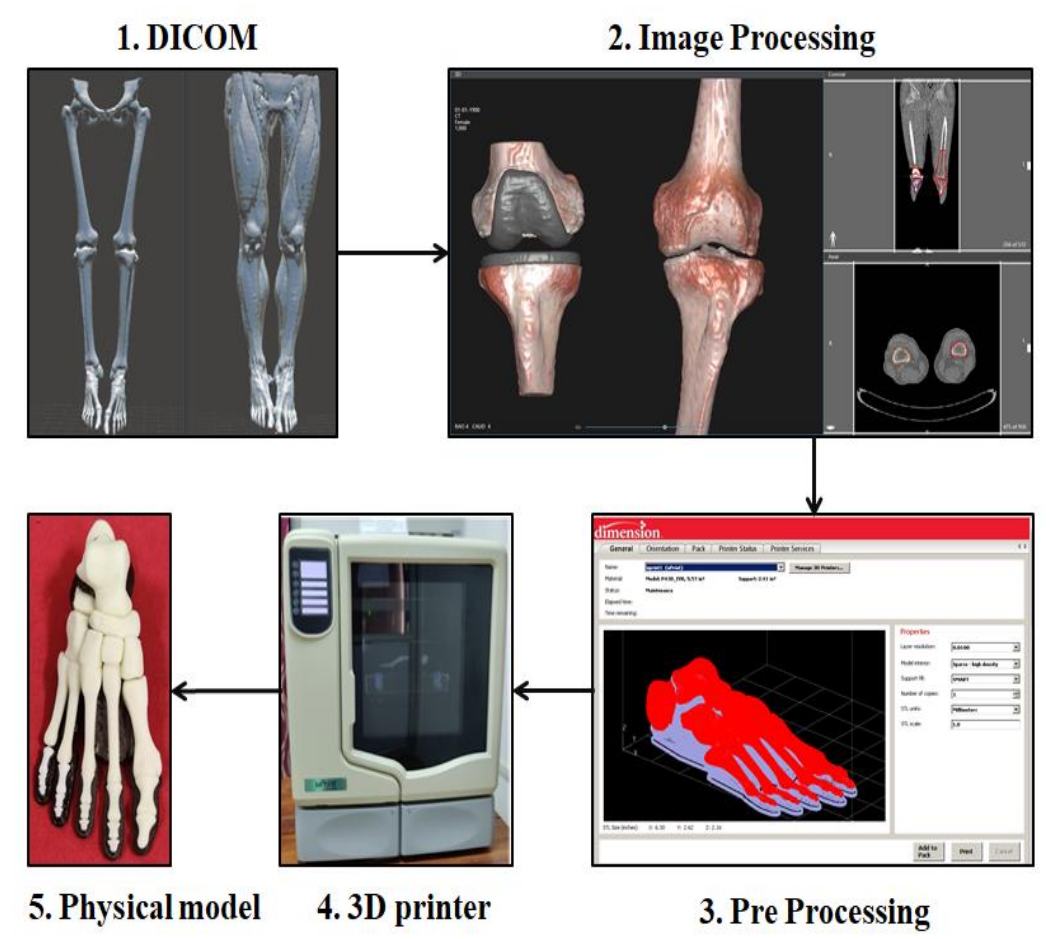

Figure 2 Fabrication steps of 3D printed model from medical imaging.

Communication with uPrint and preprocessing such as orientation, layer thickness, infill, scaling and quantity are controlled by CatalystEX software. Production of $3 \mathrm{D}$ physical object from the output file of D2P is completed by FDM based 3D printer of uPrint (Stratasys Ltd, Minnesota, Rehovot, Israel). Layer thickness of the printer is $0.254 \mathrm{~mm}$ and build volume is $203 \times 152 \times 152$ $\mathrm{mm}$ in $\mathrm{X}, \mathrm{Y}$ and $\mathrm{Z}$ planes respectively. Model material of ABS P430 and soluble support material of SR-30 is used for manufacturing the 3D printed objects. Fabrication steps of 3D printed model from medical imaging as shown in figure 2 .

\section{RESULTS}

In this study, it is evident that D2P used to segregate the complete leg bone region apart from other human anatomy parts play vital role in anatomical modeling. First and foremost, step in this process is global thresholding technique which was applied on whole CT datasets to extract bone data available from the datasets. Then region-based segmentation was performed manually to identify and segment the bone regions associated with the leg. In parallel, ankle foot of the leg also segmented to enhance the study. 
After the segmentation of ROI, meshed parts of anatomical model undergo smoothness adjustment, topological optimization and cropping to enhance the characteristics of the model that fulfill education purpose. The wall thickness of developed anatomical 3D model can be analyzed which in turn enable development of 3D printed ankle-foot model from the whole leg bone model which includes tarsals, metatarsals, and phalanges. Here, wall thickness analysis is performed to predict the weak portions of the model which need extra care and support during development process. This reduces time and contains issues faced during the printing an anatomical model. Results of the wall thickness analysis are shown in Figure 3. From this figure, weak to strong regions are indicated by the colors namely red, yellow, green and blue respectively. Then, the required file format for 3D printing process is generated by using $\mathrm{D} 2 \mathrm{P}$.
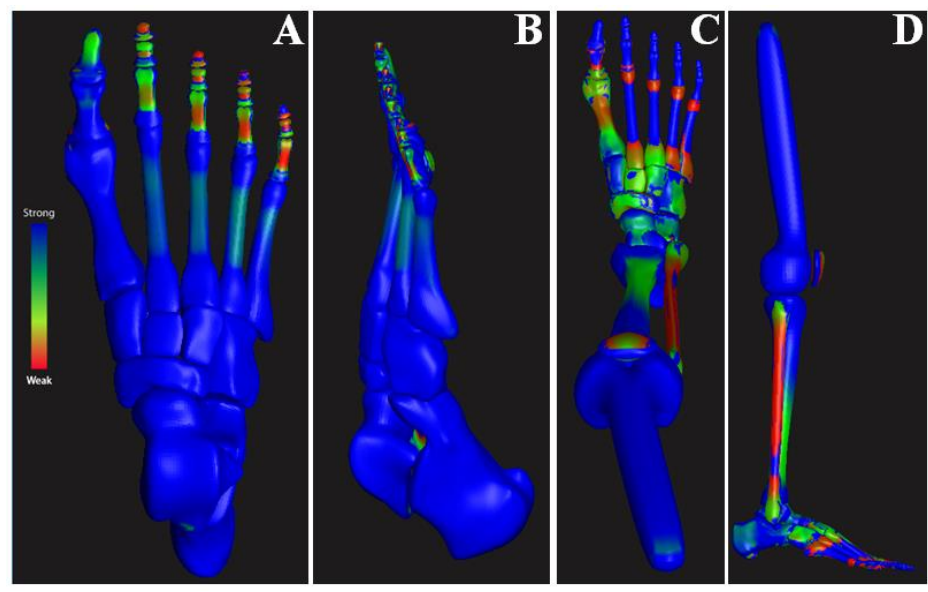

Figure 3 Results of wall thickness analysis (A) Top view of foot, (B) Side view of foot (C) Top view of leg and (D) Side view of leg.

3D printed ankle foot and leg prototypes with support material are shown in figure $4 \mathrm{~A}$ and $4 \mathrm{~B}$ respectively. For clear visualization and demonstration purpose, actual size of the ankle foot is fabricated which consists of 7 tarsals, 5 metatarsals and 14 phalanges. Due to understanding, the bone arrangement of the leg is scaled down to the dimensions of the leg model. Complete leg model consists of ankle foot, tibia, fibula, patella, and femur. A maximum dimension of the foot is $160 \times$ $67 \times 55 \mathrm{~mm}$, and the leg is $140 \times 21 \times 49 \mathrm{~mm}$. To identify the scale bar, prototypes are photographed with Indian one rupee coin. Owing to application of the models and reduction of cost both the models are printed in high density type of model fill and smart type of support fill. Similarly, for reducing the support material consumption, foot model is placed in XY orientation and leg model is placed in $\mathrm{XZ}$ orientation as shown in figure $4 \mathrm{~A}$ and $4 \mathrm{~B}$. 

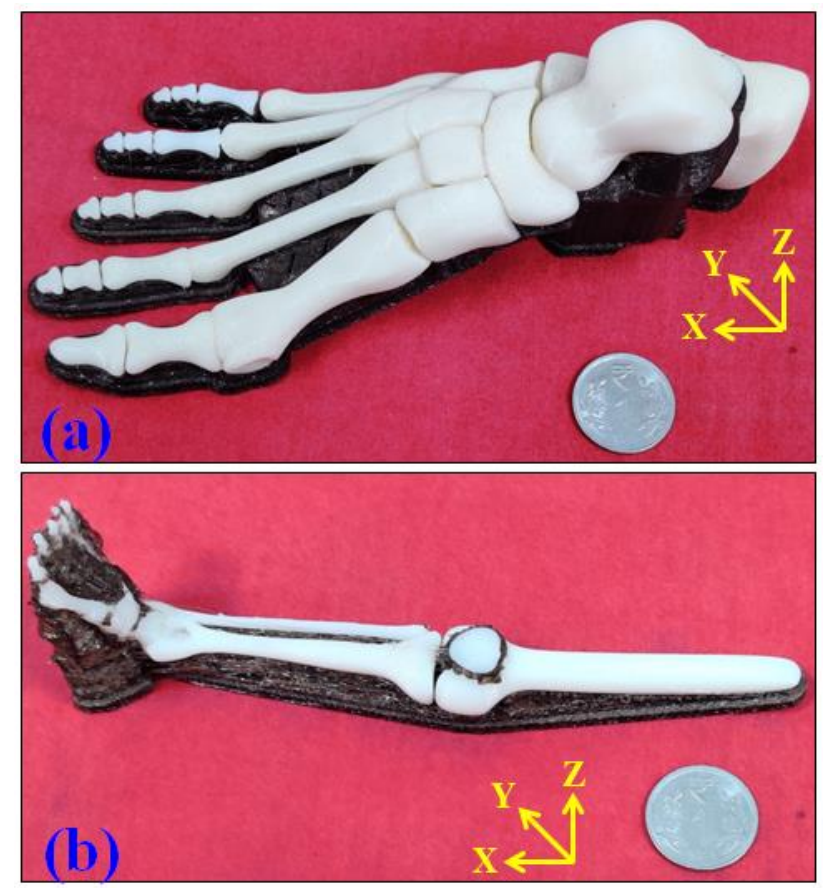

Figure 4 3D printed patient-specific (a) Ankle foot, (b) Leg.

Printing details of the 3D printed ankle foot and leg prototypes are taken from CatalystEX software. Ankle foot prototype consumes $3.22 \mathrm{in}^{3}$ model materials, $1.38 \mathrm{in}^{3}$ support materials and it takes 7.09 hours to complete the print. Similarly, printing time of the miniature leg model is 2.14 hours and it consumes $0.50 \mathrm{in}^{3}$ and $0.48 \mathrm{in}^{3}$ of model and support materials respectively. Prototyping cost of the ankle foot is INR 4,145.00 and leg is INR 1,338.00. Owing to difficulties in the manual assembly of anatomy, post processing is not necessary for this application.

\section{DISCUSSION}

Accuracy of 3D printed model depends up on the capability of 3D printer and quality of the input file provided. FDM uPrint and D2P software serves enough accuracy to fabricate the 3D printed bone models. Range of CT number used to denote various density areas on Hounsfield scale are 1000 for air, 0 for water and +1000 for bone. CT is capable of showing denser region like bone which helps to investigate skeletal anatomy and makes it predominant imaging modality over other modalities (Molteni, 2013). There are some difficulties in visualization and understand the portions covered by support material. In FDM uPrint, support materials are removed by water soluble solution named as post processing. In this application, assembly of individual bone portions is a very challenging task. In this regard, post processing is neglected to a preliminary study.

To validate the 3D printed models, feedback was collected from eleven experienced orthopedic 
surgeons who are expertise in their field in Coimbatore, Tamil Nadu, India. During the personal interaction with filed experts, they were requested to rate the developed 3D printed bone models on satisfaction rate over the cadaver models. The questionnaire includes aesthetic, accuracy, durability, comfort, time and cost of the 3D printed model. On all the foresaid aspect it satisfies the doctor's expectation. Developed model intended to undergo only wall thickness analysis rather than FEA (Finite Element Analysis). Indeed, FEA would involve time consuming tedious process used mainly to assess functional outcome of the model. Where, objective of developed model was to implement on education and training in place of cadaver. Therefore, wall thickness analysis is quite enough for this study to print the anatomical model.

Results of the data collection infer that; 3D printed models are alterative for traditional techniques. The development time and cost of 3D printed anatomical models are less when compared to the cadaver models. Developed 3D printed patient-specific ankle foot is $31.05 \%$ cheaper when compared to cadaver models. Total development time is calculated by addition of time required for image processing and 3D printing. Here, segmentation of bone models consumes 2 hours and fabrication takes 10 hours. Noticeably, image processing time is based on skill of the expertise using software, system configuration, quality, and complexity of DICOM file. Similarly, influencing process parameters, type of 3D printers and its technology can vary the printing time. The biocompatible material is also available in 3D printing; sterilization of the product is also easy.

\section{CONCLUSION}

This study gives a proof of concept to use image processing and 3D printing techniques together effectively produce the 3D printed patient-specific model for anatomy education. The developed models offer the instant solution and reuse, which prominently increase the standard of medical education with affordable cost. Moreover, 3D printed models can improve the skill, confident and proficient of the medical residents to increase their success rate by augmenting the educational method. The proposed framework for developing 3D printed patient-specific models provides identical benefits to medical students and experts to demonstrate the pre-operative planning and surgical training instead of practicing on cadaver model. Although, ethical issue, lack of biomechanical properties, biocompatibility and difficulties in segmentation and design stage is the shortcomings of these techniques. However, improvements in AM techniques such as multimaterial, multi-color and organ printing will play a vital role in healthcare sectors. 


\section{ACKNOWLEDGMENT}

We thank Centre of Excellence in Manufacturing Sciences (CoEMS) at Coimbatore Institute of Technology, India for the support and technical assistance.

\section{REFERENCES}

AbouHashem, Y. Dayal, M. Savanah, S. \& Štrkalj, G. 2015. The application of 3D printing in anatomy education. Medical Education Online 20 (1): 1-4.

Aimar, A. Palermo, A. \& Innocenti, B. 2019. The Role of 3D Printing in Medical Applications: A State of the Art. Journal of Healthcare Engineering 2019.

Bibb, R. Eggbeer, D. \& Paterson, A. 2015. Working with medical scan data. In Medical Modelling: The Application of Advanced Design and Rapid Prototyping Techniques in Medicine (Second, pp. 35-63). Elsevier Ltd.

Bidgood Jr WD, Horii SC, Prior FW, V. S. DE. 1997. Understanding and Using DICOM, the Data Interchange Standard for Biomedical Imaging. Journal of the American Medical Informatics Association 4 (3): 199-212.

Byrne, N. Velasco Forte, M. Tandon, A. Valverde, I. \& Hussain, T. 2016. A systematic review of image segmentation methodology, used in the additive manufacture of patient-specific 3D printed models of the cardiovascular system. JRSM Cardiovascular Disease 5 204800401664546.

Chang, D. Tummala, S. Sotero, D. Tong, E. Mustafa, L. Mustafa, M. Browne, W. F. \& Winokur, R. S. 2019. Three-Dimensional Printing for Procedure Rehearsal/Simulation/Planning in Interventional Radiology. Techniques in Vascular and Interventional Radiology 22 (1): 14-20.

Chen, S. Pan, Z. Wu, Y. Gu, Z. Li, M. Liang, Z. Zhu, H. Yao, Y. Shui, W. Shen, Z. Zhao, J. \& Pan, H. 2017. The role of three-dimensional printed models of skull in anatomy education: A randomized controlled trail. Scientific Reports 7 (1): 1-11.

Drapkin, Z. A. Lindgren, K. A. Lopez, M. J. \& Stabio, M. E. 2015. Development and assessment of a new 3D neuroanatomy teaching tool for MRI training. Anatomical Sciences Education 8 (6): 502-509.

Estevez, M. E. Lindgren, K. A. \& Bergethon, P. R. 2010. A novel three-dimensional tool for teaching human neuroanatomy. Anatomical Sciences Education 3 (6): 309-317. 
Fan, J. Zeng, G. Body, M. \& Hacid, M. S. 2005. Seeded region growing: An extensive and comparative study. Pattern Recognition Letters 26 (8): 1139-1156.

Ganguli, A. Pagan-Diaz, G. J. Grant, L. Cvetkovic, C. Bramlet, M. Vozenilek, J. Kesavadas, T. \& Bashir, R. 2018. 3D printing for preoperative planning and surgical training: a review. Biomedical Microdevices 20 (3).

Govsa, F. Ozer, M. A. Sirinturk, S. Eraslan, C. \& Alagoz, A. K. 2017. Creating vascular models by postprocessing computed tomography angiography images: a guide for anatomical education. Surgical and Radiologic Anatomy 39 (8): 905-910.

Hnatkova, E. Kratky, P. \& Dvorak, Z. 2014. Conversion of 2D medical scan data into 3D printed models. Advances in Environmental Sciences, Development and Chemistry 315-318.

Iancu, C. 2018. About 3D Printing File Formats. Annals of the Constantin Brancusi University of Targu Jiu-Letters \& Social Sciences Series 1 (2): 135-138.

Li, K.H.C., Kui, C., Lee, E.K.M., Ho, C.S., Sunny Hei, S.H., Wu, W., Wong, W.T., Voll, J., Li, G., Liu, T. and Yan, B. 2017. The role of 3D printing in anatomy education and sur gical tr aining: A narrative review. MedEdPublish 6 (2): 1-23.

Lim, K. H. A. Loo, Z. Y. Goldie, S. J. Adams, J. W. \& McMenamin, P. G. 2016. Use of 3D printed models in medical education: A randomized control trial comparing 3D prints versus cadaveric materials for learning external cardiac anatomy. Anatomical Sciences Education 9 (3): 213-221.

Molteni, R. 2013. Prospects and challenges of rendering tissue density in Hounsfield units for cone beam computed tomography. Oral Surgery, Oral Medicine, Oral Pathology and Oral Radiology 116 (1): 105-119.

Mukul Ramola, Vinod Yadav, R. J. 2018. On the adoption of additive manufacturing in healthcare : a literature review. Journal of Manufacturing Technology Management 30 (1): 4869.

Ozturk, A. M. Suer, O. Coban, I. Ozer, M. A. \& Govsa, F. 2020. Three-Dimensional Printed Anatomical Models Help in Correcting Foot Alignment in Hallux Valgus Deformities. Indian Journal of Orthopaedics 54 (0123456789): 199-209.

Panesar, S. S. Magnetta, M. Mukherjee, D. Abhinav, K. Branstetter, B. F. Gardner, P. A. Iv, M. \& Fernandez-Miranda, J. C. 2019. Patient-specific 3-dimensionally printed models for 
neurosurgical planning and education. Neurosurgical Focus 47 (6): 1-11.

Ratinam R, Quayle M, Crock J, Lazarus M, Fogg Q, M. P. 2019. Challenges in creating dissectible anatomical 3D prints for surgical teaching. Journal of Anatomy 234 (4): 419-437.

Smith, C. F. Tollemache, N. Covill, D. \& Johnston, M. 2018. Take away body parts! An investigation into the use of 3D-printed anatomical models in undergraduate anatomy education. Anatomical Sciences Education 11 (1): 44-53.

Soltani-Nabipour, J. Khorshidi, A. \& Noorian, B. 2020. Lung tumor segmentation using improved region growing algorithm. Nuclear Engineering and Technology 52 (10): 2313-2319.

Starosolski, Z. A. Kan, J. H. Rosenfeld, S. D. Krishnamurthy, R. \& Annapragada, A. 2014. Application of 3-D printing (rapid prototyping) for creating physical models of pediatric orthopedic disorders. Pediatric Radiology 44 (2): 216-221.

Tracey, J. Arora, D. Gross, C. E. \& Parekh, S. G. 2019. Custom 3D-Printed Total Talar Prostheses Restore Normal Joint Anatomy Throughout the Hindfoot. Foot and Ankle Specialist 12 (1): $39-48$.

Van Eijnatten, M. Berger, F. H. De Graaf, P. Koivisto, J. Forouzanfar, T. \& Wolff, J. 2017. Influence of CT parameters on STL model accuracy. Rapid Prototyping Journal 23 (4): 678685.

Van Eijnatten, M. van Dijk, R. Dobbe, J. Streekstra, G. Koivisto, J. \& Wolff, J. 2018. CT image segmentation methods for bone used in medical additive manufacturing. Medical Engineering and Physics 51 6-16.

Van Epps, A. Huston, D. Sherrill, J. Alvar, A. \& Bowen, A. 2015. How 3D Printers Support Teaching in Engineering, Technology and Beyond. Bulletin of the American Society for Information Science and Technology 42 (1): 16-20.

Vithani, K. Goyanes, A. Jannin, V. Basit, A. W. Gaisford, S. \& Boyd, B. J. 2019. An Overview of 3D Printing Technologies for Soft Materials and Potential Opportunities for Lipid-based Drug Delivery Systems. Pharmaceutical Research 36 (1).

Wallner, J. Hochegger, K. Chen, X. Mischak, I. Reinbacher, K. Pau, M. Zrnc, T. SchwenzerZimmerer, K. Zemann, W. Schmalstieg, D. \& Egger, J. 2018. Clinical evaluation of semiautomatic open-source algorithmic software segmentation of the mandibular bone: Practical feasibility and assessment of a new course of action. PLoS ONE 13 (5): 1-26. 
Yoo, D. J. 2011. Three-dimensional surface reconstruction of human bone using a B-spline based interpolation approach. CAD Computer Aided Design 43 (8): 934-947.

Zhang, J. \& Li, Y. 2016. Region segmentation and shape characterisation for tessellated CAD models. International Journal of Computer Integrated Manufacturing 29 (8): 907-915. 